\title{
Atypical Presentation of Acanthamoeba Keratitis in a Contact Lens Wearer
}

\author{
Nada Al-Yousuf ${ }^{1}$, Khalid Al Sindi ${ }^{2}$, Shaima Hashem ${ }^{3}$, Hasan Alsetri ${ }^{4}$, Catherine Dombroski ${ }^{4}$ \\ ${ }^{1}$ Department of Ophthalmology, King Abdullah Medical City, Arabian Gulf University, Manama, Kingdom of Bahrain \\ ${ }^{2}$ Departments of Pathology, Blood Bank and Laboratory Medicine, King Hamad University Hospital, Muharraq, \\ Kingdom of Bahrain \\ ${ }^{3}$ Department of Ophthalmology, Salmaniya Medical Complex, Manama, Kingdom of Bahrain \\ ${ }^{4}$ Department of Chemistry and Biochemistry, University of California, Los Angeles, CA, USA \\ Email: nyousuf10@gmail.com
}

How to cite this paper: Al-Yousuf, N., Al Sindi, K., Hashem, S., Alsetri, H. and Dombroski, C. (2020) Atypical Presentation of Acanthamoeba Keratitis in a Contact Lens Wearer. Open Journal of Ophthalmology, 10, 261-267.

https://doi.org/10.4236/ojoph.2020.104028

Received: July 12, 2020

Accepted: October 19, 2020

Published: October 22, 2020

Copyright (c) 2020 by author(s) and Scientific Research Publishing Inc. This work is licensed under the Creative Commons Attribution International License (CC BY 4.0).

http://creativecommons.org/licenses/by/4.0/

\section{(c) (i) Open Access}

\begin{abstract}
Acanthamoeba keratitis is a serious infection that can lead to loss of vision. It is highly challenging and often poses a diagnostic dilemma, causing delay in diagnosis and treatment. We report herewith the clinical and histopathology findings of a patient with an atypical presentation of acanthamoeba keratitis in Bahrain. The patient is a 16-year-old Bahraini teenager who was a cosmetic contact lens wearer. She presented with clinical signs and symptoms of microbial keratitis, which was initially misdiagnosed elsewhere as a case of herpetic corneal infection. Her corneal biopsy confirmed the clinical diagnosis as acanthamoeba keratitis. The patient was started on anti amoebic treatment. The infection got eradicated. The cornea healed with a central scar. Eventually, she underwent penetrating keratoplasty. This case report serves to raise awareness of this rare condition. Clinicians should have a high index of suspicion when diagnosing such cases among contact lens wearers. Early diagnosis and treatment are crucial to prevent serious complications.
\end{abstract}

\section{Keywords}

Acanthamoeba, Keratitis, Contact Lens, Perineuritis, Hypopyon

\section{Introduction}

Acanthamoeba keratitis (AK) is a sight-threatening infection of the cornea by amoebae of the genus Ancanthamoeba [1]. It is a rare disease with an estimated incidence of 1.2 per million in regular populations and 18.84 per million in contact lens wearers [2]. Documentation and awareness of this rare disease are im- 
portant as patients have the potential to lose their vision if not diagnosed and treated early [1] [3].

Contact lens (CL) wear is the most common risk factor for AK [4], however, the disease can also be present in non CL wearers at a lower incidence rate [5]. Obtaining a detailed medical history, as well as CL care routine, namely the use of tap water with homemade saline to clean the CLs, is crucial to suspect the diagnosis. Treatment history can be helpful in the diagnosis when patients get treated for herpetic keratitis with no improvement. This leads to considering AK as a possibility. Common symptoms associated with AK include foreign body sensation, severe ocular pain, photophobia, excessive tearing and blurring of vision. The pain is typically described as out of proportion to the objective clinical findings [6]. Slit-lamp examinations reveal the stage of the disease's progression. Epitheliitis occurs early in the disease, characterized by punctate keratitis, elevated epithelial lines, or dendriform irregularities. As the disease progresses, radial neuritis is observed, followed by anterior stromal disease, which progresses to deep stromal keratitis, or ring infiltrate [6]. The classic ring infiltrate is seen in approximately $50 \%$ of AK patients [6] [7]. Laboratory diagnosis via visualization of the amoeba in corneal scrapings or corneal biopsies is a reliable diagnostic method. In severe cases of $\mathrm{AK}$, amoebae density can be very high, making detection of the amoeba via direct microscopy easy in $200-400$ magnification of the original sample. Acanthamoeba trophozoites or cysts can easily be detected using methods such as phase contrast microscopy, as well as staining and fluorometry, as cysts exhibit auto-fluorescence [3]. The treatment of AK can be challenging, as the amoeba's cyst form is highly resistant to therapy. A combination of treatments is generally used. Chlorhexidine $0.02 \%$ combined with $0.1 \%$ propamidine, or polyhexamethylene biguanide (PHMB) $0.02 \%$ combined with hexamidine (Desmodine) $0.1 \%$ [3] [4]. Addition of topical voriconazole to the above regimen was used with success in AK treatment [8]. Moreover, oral voriconazole combined with topical PHMB was shown clinically to be a successful treatment [9]. If AK is not detected early and treated appropriately, it can lead to loss of vision [10]. The earlier the diagnosis is, the better the prognosis will be. In some patients, corneal graft surgery is required to eradicate the infection, restore vision, and prevent eye loss [3] [7].

The purpose of this paper is to present a patient with $\mathrm{AK}$ who was initially misdiagnosed with herpetic keratitis. She has some clinical findings that are not typical of AK. This case directs the attention of clinicians towards diagnosing AK even with atypical clinical findings.

\section{Case Report}

The patient is a 16-year-old Bahraini female who wore colored cosmetic contact lenses for several weeks prior to her presentation to our practice. She presented with right eye pain, redness, photophobia, watering, foreign body sensation and reduction in vision. She admitted to a pattern of sleeping with the contact lenses. 
She had a history of sickle cell trait and reduced glucose-6-phosphate dehydrogenase (G6PD) activity. She was initially treated for bacterial keratitis but later treated for herpetic keratitis for which she received a course of acyclovir. When the patient did not improve, she was referred to our practice. On presentation, her vision was hand motion (HM) in the right eye and 20/20 in the left eye. She had reduced corneal sensation in the right eye. Slit lamp examination was normal in the left eye. The right eye showed radial perineuritis and multifocal nummular stromal infiltrates (Figure 1). Anterior chamber examination showed a steak of hypopyon. There was no ring infiltrate. Fundus examination was normal.

Acanthamoeba keratitis was suspected. Corneal biopsy was performed and the histopathology report revealed a foamy cell nested between corneal epithelial cells consistent with Acanthamoeba trophozoites (Figure 2). The patient was started on $0.1 \%$ propamidine isethionate (hexamidine) eye drops and $0.02 \%$ chlorhexidine eye drops hourly. Fortified voriconazole eye drops were administered around the clock in the first 48 hours, then hourly during daytime for the next 3 days, then tapered to every 4 hours. Oral ketoconazole $400 \mathrm{mg}$ was administered once daily and preservative-free lubricant eye drops were applied every 4 hours.

Hypopyon was resolved gradually. The patient showed the improvement of the stromal infiltration over an 8-week period. Topical voriconazole and oral ketoconazole were discontinued. Combination therapy of hexamidine and chlorhexidine in addition to preservative-free lubricant was continued for 6 months with regular monthly follow-ups. The pain settled and the keratitis healed with permanent scarring and corneal vascularization (Figure 3). Vision in the right eye was Hand Motion and a central stromal scar was present after one year follow-up. Prognosis was shown to be favorable after penetrating keratoplasty [11]. Right eye penetrating keratoplasty was performed one year after her initial presentation (Figure 4). Her condition settled well post-operatively and the corneal graft was clear. Her vision one year post-keratoplasty was 20/40 with correction.

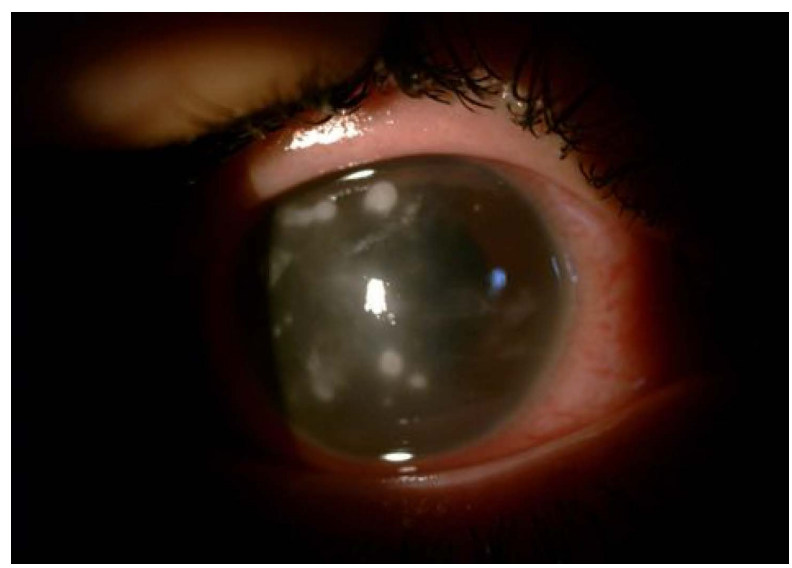

Figure 1. Slit lamp photo of the patient's right eye showing scattered round shaped keratitis patches involving the corneal stroma. 


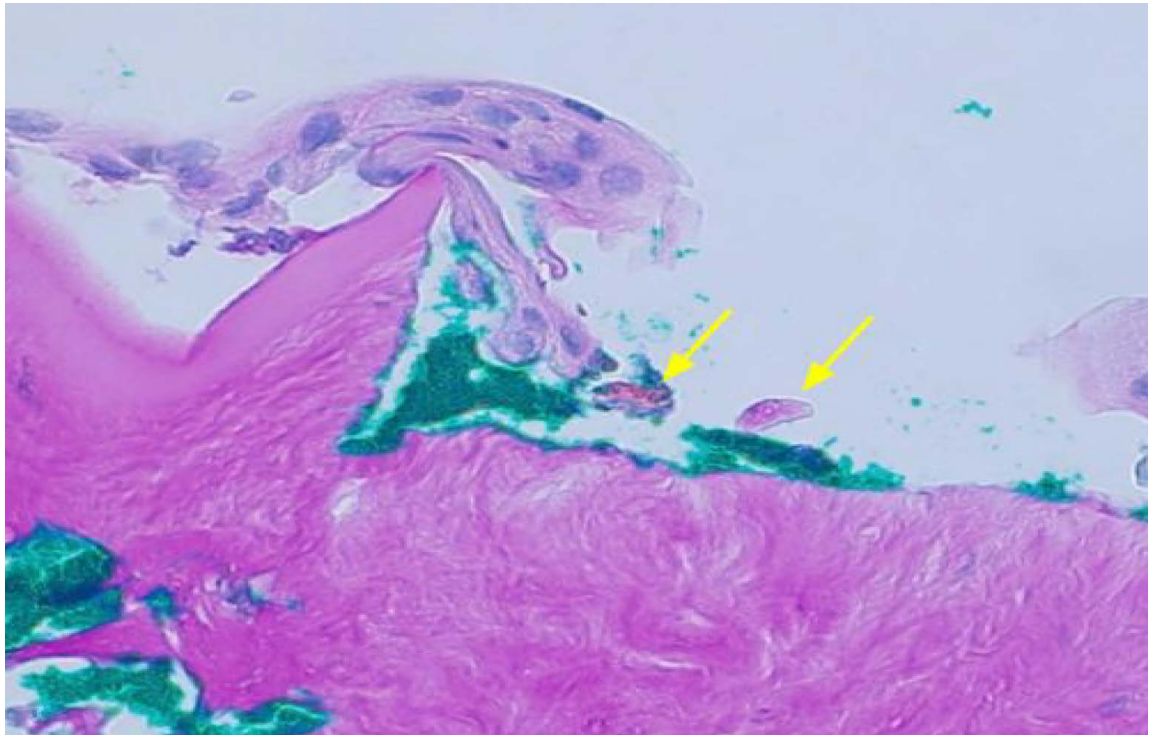

Figure 2. Acanthamoeba trophozoite highlighted by special Periodic Acid-Schiff chemical stain (arrows). Magnification power $50 \mu \mathrm{m}$.

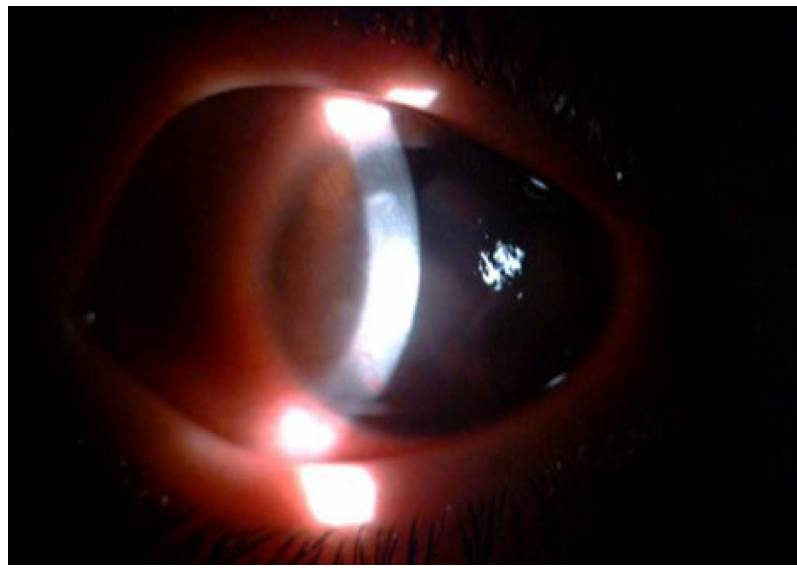

Figure 3. Slit lamp photo of the patient's right eye showing corneal scarring with vascularization.

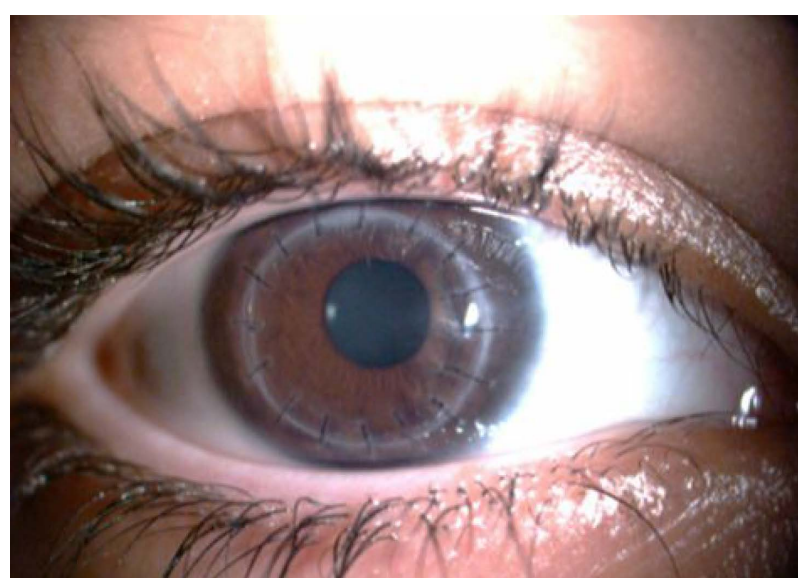

Figure 4. Slit lamp photo of the patient's right eye showing clear corneal graft. 


\section{Discussion}

Acanthamoeba keratitis (AK) is usually diagnosed in the later stages of the disease's progression. The patient was initially misdiagnosed elsewhere with stromal herpetic keratitis, a common misdiagnosis of AK in the literature [12] [13] [14].

In a review of AK, Szentmary et al. found that around $75 \%$ to $90 \%$ of all patients are misdiagnosed during the early stages of the disease [15]. Herpes keratitis is a common misdiagnosis of $\mathrm{AK}$, since both disorders show stromal necrosis, as well as negative microbiology cultures. This patient in particular had reduced corneal sensation, which contributed to her initial misdiagnosis of herpetic keratitis by the clinician that first received her. Although the reduction of corneal sensation is typical in herpetic keratitis, clinicians should not be misled by this symptom, because it is also a common occurrence in chronic contact lens wearers [6].

This patient is unique from most reported $\mathrm{AK}$ patients due to the absence of typical AK signs, particularly the stromal ring infiltrate. A clinical review of Acanthamoeba Keratitis showed that ring infiltration is one of the common signs [15].

A recent report supports this [16]. Another unusual clinical feature in this case is the presence of hypopyon. In a review of AK, Lorenzo-Morales et al. did not cite hypopyon among the clinical features of the disease [3]. Additionally, Szentmary et al. reported that hypopyon is a very rare clinical sign [15].

Acanthamoeba keratitis is commonly a late diagnosis for several reasons. Clinicians may not consider this infection in the differential diagnoses because of its rarity. AK is often treated as bacterial as well as herpetic keratitis and is only considered after the patient does not respond to treatment for an extended period of time. In addition, the vast majority of AK patients are contact lens wearers who typically seek medical help later than usual because they are accustomed to CL-associated irritations. Moreover, clinicians may miss this disease because of its atypical features and/or lack of some typical signs.

This patient is a CL wearer, therefore, AK should be a consideration, as CL wearers have an increased probability of having an $\mathrm{AK}$ infection. She is a teenager and was dispensed a cosmetic CL. Teenagers should only be prescribed CLs for optical and medical conditions such as keratoconus, persistent epithelial defects and other conditions that necessitate CLs. Furthermore, these individuals should be supervised to ensure particular lens care and hygiene. All CLs, including cosmetic ones, should only be prescribed by healthcare providers and should not be available over-the-counter [17] [18]. Cosmetic contact lenses in particular should be contraindicated to underaged individuals.

Prognosis is more favorable in cases that are diagnosed and treated early. Early treatment may result in the eradication of the parasitic organism, preventing the need for corneal graft surgery. Therefore, collaboration with a microbiologist is important to improve the prognosis and to prevent complications [17] [18]. 
Although this patient developed corneal scarring, she had good visual outcome after a penetrating keratoplasty procedure. This procedure is supported by Kitzmann et al. who demonstrated that penetrating keratoplasty performed for patients with corneal scarring, was associated with good visual prognosis [11].

\section{Conclusion}

Clinicians should consider the possibility of AK in all CL wearers. Absence of typical features of AK such as ring infiltrate should not dismiss the disease as a possibility. Even when confronted with atypical presentations, clinicians should maintain a high index of suspicion towards the possibility of AK. Tissue diagnosis and collaboration with a microbiologist to diagnose the condition is important for early treatment and to prevent complications.

\section{Conflicts of Interest}

The authors declare no conflicts of interest regarding the publication of this paper.

\section{References}

[1] Marciano-Cabral, F. and Cabral, G. (2003) Acanthamoeba spp. as Agents of Disease in Humans. Clinical Microbiology Reviews, 16, 273-307. https://doi.org/10.1128/CMR.16.2.273-307.2003

[2] Radford, C.F., Minassian, D.C. and Dart, J.K. (2002) Acanthamoeba Keratitis in England and Wales Incidence, Outcome, and Risk Factors. British Journal of Ophthalmology, 86, 536-542. https://doi.org/10.1136/bjo.86.5.536

[3] Lorenzo-Morales, J., Khan, N.A. and Walochnik, J. (2015) An Update on Acanthamoeba Keratitis: Diagnosis, Pathogenesis and Treatment. Parasite, 22, 1-20 https://doi.org/10.1051/parasite/2015010

[4] Seal, D.V. (2003) Acanthamoeba Keratitis Update Incidence, Molecular Epidemiology and New Drugs for Treatment. Eye, 17, 893-905. https://doi.org/10.1038/sj.eye.6700563

[5] Chynn, E.W., Lopez, M.A, Pavan-Langston, D. and Talamo, J.H. (1995) Contact Lens and Non Contact Lens Characteristics. Ophthalmology, 102, 1369-1373. https://doi.org/10.1016/S0161-6420(95)30862-7

[6] Krachmer, J. Mannis, M. and Holland, E. (2010) Cornea: 3rd Edition. Elsevier, Amsterdam.

[7] Moore, M.B. and McCulley, J.P. (1989) Acanthamoeba Keratitis Associated with Contact Lenses: Six Consecutive Cases of Successful Management. British Journal of Ophthalmology, 73, 271-275. https://doi.org/10.1136/bjo.73.4.271

[8] Masselam, K. and Pineda, R. (2008) Topical Voriconazole as an Effective Co-Agent against Acanthamoeba Keratitis. Investigative Ophthalmology \& Visual Science, 49, 5537

[9] Hou, T., Chen, Y. and Hsu, C. (2017) Rapid Resolution of Stromal Keratitis with the Assistance of Oral Voriconazole in Resistant Acanthamoeba Keratitis. Taiwan Journal of Ophthalmolgy, 7, 224-226. https://doi.org/10.4103/tjo.tjo 7317

[10] Bacon, A.S., Frazer, D.G., Dart, J.K., Matheson, M., Ficker, L.A. and Wright, P. (1993) A Review of 72 Consecutive Cases of Acanthamoeba Keratitis, 1984-1992. 
Eye, 7, 719-725. https://doi.org/10.1038/eye.1993.168

[11] Kitzmann, A.S., Goins, K.M., Sutphin, J.E. and Wagoner, M.D. (2009) Keratoplasty for Treatment of Acanthamoeba Keratitis. Ophthalmology, 116, 864-869.

https://doi.org/10.1016/j.ophtha.2008.12.029

[12] Perry, H.D., Donnenfeld, E.D., Foulks, G.N., Moadel, K. and Kanellopoulos, A.J. (1995) Decreased Corneal Sensation as an Initial Feature of Acanthamoeba Keratitis. Ophthalmology, 102, 1565-1568.

https://doi.org/10.1016/S0161-6420(95)30830-5

[13] Laerhout, I., Goegebuer, A., Van Den, B.C. and Kestelyn, P. (2004) Delay in Diagnosis and Outcome of Acanthamoeba Keratitis. Graefe's Archive for Clinical and Experimental Ophthalmology, 242, 648-653. https://doi.org/10.1007/s00417-003-0805-7

[14] Stan, C., Cristina, V. and Mihael, P. (2016) Acanthamoeba Keratitis Challenges a Case Report. Romanian Journal of Ophthalmology, 60, 40-42.

[15] Szentmary, N., Daas, L., Shi, L., Laurik, K.L., Milioti, G., Seitz, B., et al. (2019) Acanthamoeba Keratitis-Clinical Signs, Differential Diagnosis and Treatment. Journal of Current Ophthalmology, 31, 16-23.

https://doi.org/10.1016/j.joco.2018.09.008

[16] Agab, A., Albuainain, A. and Al Bin Ali, G. (2017) Unilateral Acanthamoeba Keratitis Following the Use of Cosmetic Contact Lens. Bahrain Medical Bulletin, 39, 69-71. https://doi.org/10.12816/0047447

[17] Drancourt, M. and Hoffart, L. (2017) Infectious Diseases. 4th Edition, Elsevier Ltd., Amsterdam.

[18] Dini, L.A., Cockinos, C., Frean, C., Niszi, I.A. and Markus, M.B. (2000) Unusual Case of Acanthamoeba polyphaga and Pseudomonas aeruginosa Keratitis in a Contact Lens Wearer. Journal of Clinical Microbiology, 38, 826-829.

https://doi.org/10.1128/JCM.38.2.826-829.2000 